Voix et Images

\title{
Focalisation, voyeurisme et scène originaire dans Serge d'entre les morts
}

\section{Alain Piette}

Volume 12, numéro 3 (36), printemps 1987

Yves Beauchemin

URI : https://id.erudit.org/iderudit/200662ar

DOI : https://doi.org/10.7202/200662ar

Aller au sommaire du numéro

Éditeur(s)

Université du Québec à Montréal

ISSN

0318-9201 (imprimé)

1705-933X (numérique)

Découvrir la revue

Citer cet article

Piette, A. (1987). Focalisation, voyeurisme et scène originaire dans Serge d'entre les morts. Voix et Images, 12(3), 497-511. https://doi.org/10.7202/200662ar d'utilisation que vous pouvez consulter en ligne.

https://apropos.erudit.org/fr/usagers/politique-dutilisation/ 


\title{
Focalisation, voyeurisme et scène originaire dans Serge d'entre les morts 1
}

\author{
par Alain Piette, Université du Québec à Montréal \\ A Henri-Paul Jacques \\ la voir ah la voir \\ la voir oui la voir \\ (Serge d'entre les morts, p. 23 et 122)
}

Variations sur un même thème, effets stylistiques divergents jouant sur l'infinitésimal, à moins qu'il ne s'agisse de simples répétitions compulsives. Tout compte fait, les trois hypothèses sont peut-être pertinentes et mon propos ne sera donc pas ici de trancher la question. Je tenterai plutôt de décrire le phénomène par lequel ce beau texte se répète à la façon d'un Boléro de Ravel inusité: la notion de focalisation permet en effet d'analyser la représentation des perceptions en général et de la perception visuelle en particulier. Mais l'étude de la focalisation est-elle un simple outil descriptif? N'ouvre-t-elle pas la voie à une "narratologie critique" pour reprendre l'heureuse formulation de Mieke Bal2? Ainsi il ne faudra pas se limiter à répertorier des phénomènes, fût-ce de façon très minutieuse, mais cette classification même devra servir à une meilleure compréhension du texte et de ses enjeux.

Le choix du présent corpus nécessite enfin quelques mots d'explication. Dans ce roman, tout est sensation, désir, intuition. Tous les sens sont activés ${ }^{3}$. J'endosse entièrement cet énoncé de Donald Smith qui circonscrit on ne peut mieux l'objet de ma recherche. Mais tout pertinent qu'il soit, ce projet apparaît ambitieux ou inutile si on le confronte à la lecture de Gérard Bessette dans le Semestre ${ }^{4}$. N'y a-t-il pas là, outre la psychocritique de Serge d'entre les morts, quelques pages suir l'optique de ce romañ (ce qui recoupe le terme «focalisation»)? Plutôt que de ratiociner là-dessus, je préfère reconnaître d'emblée ma dette envers le texte stimulant de Bessette dont certains acquis sont incontestables: j'essaierai d'éviter le double emploi et, je l'espère, de pousser l'investigation plus loin, là où le commentaire bessettien se fait plus laconique (pour ne pas dire lacunaire). En définitive, dans le Semestre, il s'agit bien d'un pacte romanesque. Peut-on recevoir sans distanciation le travail de Sandra sur l'optique dans Serge d'entre les morts? Et si Bessette s'est visiblement amusé par moments dans une auto-biographie réelle (Mes romans et moi), que n'en sera-t-il pas d'une autobiographie fictive? Alors sans plus tarder, écarter le rideau, assez discrètement pour ne pas être vu, mais assez habilement pour voir... 
J'ai parlé de focalisation en présupposant la notion connue du lecteur. Gérard Genette l'a introduite en 1972 dans Figure III, à partir de l'expression de Brooks et Warren "focus of narration", pour désigner ce qu'on appelle ailleurs "perspective», "point de vue», "optique», "vision" ou "champ" 6 . Il entendait par là se soustraire à ce qu'il y a de trop spécifiquement visuel dans les autres termes mais "focalisation" malgré son suffixe abstrait n'élimine pas vraiment le rapport au visuel que l'on trouve dans «focal» et "focaliser". Ainsi l'ensemble des perceptions n'est pas recouvert par ce terme et c'est bien pourtant l'objet visé par Genette ainsi qu'il le définit dans le Nouveau discours du récit ${ }^{7}$, mais sans citer Bal qui était pourtant responsable de cet élargissement ${ }^{8}$. Toutefois, la difficulté de trouver un terme adéquat me rallie à la position de Bal elle-même et je parlerai donc de focalisation en sachant bien qu'il s'agit là de l'ensemble des perceptions. Cela correspond d'ailleurs à une certaine prépondérance de la perception visuelle sur les autres perceptions dans la représentation des textes narratifs.

La nouveauté et l'audace de l'apport genettien résidait surtout dans sa distinction entre les deux questions Qui voit? (Qui perçoit?) et Qui parle?. C'était là reconnaître implicitement deux instances différentes, conclusion que n'a pas manqué de tirer Mieke Bal dans sa Narratologie ${ }^{9}$. Cependant, dans le Nouveau discours du récit, Genette fait marche arrière de façon étonnante en ne voulant à aucun prix consacrer l'autonomie de l'instance focalisatrice ${ }^{10}$. S'il est si évident qu'on ne doit pas confondre celui qui parle et celui qui perçoit, comment peut-on en arriver à ne pas poser deux sujets distincts, un sujet de parole et un sujet de perception? "Instance" ne signifie pas plus "personne humaine" dans un cas que dans l'autre! Je réponds ici à la critique de Genette dans Nouveau discours du récit (p. 43 et 48); mon impatience (le point d'exclamation) vient surtout du fait qu'il confond luimême les instances, celles de l'auteur et du narrateur:

Par focalisation, j'entends donc bien une restriction de "champ", c'est-à-dire en fait une sélection de l'information narrative par rapport à ce que la tradition nommait l'omniscience, terme qui, en fiction pure, est, littéralement absurde (l'auteur n'a rien à «savoir», puisqu'il invente tout) et qu'il vaudrait mieux remplacer par information complète - muni de quoi c'est le lecteur qui devient "omniscient".

Qu'est devenu le "narrateur omniscient" de Figures III? La mode aidant (les exclusions aussi), le couple «narrateur/narrataire» de Figures III a été évincé par l'autre couple (le vieux couple, je dirais), «auteur/lecteur». Mais à cette époque-là (1972), il ne fallait prononcer le mot "auteur" que dans un tremblement comme les Juifs de l'Ancien Testament pour le nom de Yahvé! Beaucoup d'eau a coulé sous les ponts de Paris depuis ce temps: le développement de la sociocritique et de la théorie de la réception, l'intérêt croissant pour le problème de la parodie et, il faut bien le dire, la mort de Barthes et, plus récemment, de Foucault dont l'acharnement contre la notion d'auteur "1, compréhensible et même indispensable à l'époque au plan idéologique, ne représente pas beaucoup plus maintenant que le vestige d'une vieille polémique. Mais réintroduire n'est pas confondre et Genette ferait 
bien de démêler l'écheveau de ses instances (voir aussi Nouveau discours du récit, p. 68-69 et 73).

Enfin, les deux instances (narration et focalisation) ont leurs embrayeurs spécifiques. Gérald Prince a démontré toute la complexité du processus d'attribution de la parole dans les textes narratifs ${ }^{12}$. La même entreprise pourrait être tentée pour les embrayeurs de focalisation (les verbes de perception entre autres), à cette réserve près qu'ils sont souvent implicites et donc plus difficiles à détecter.

Gérard Genette proposait une typologie à trois termes pour rendre compte des différents types de focalisations: focalisation zéro, interne et externe. Mieke Bal a procédé à une critique de cette typologie en démontrant brillamment que l'opposition entre les deux premiers termes concerne le sujet focalisateur alors que l'opposition entre les deux derniers envisage plutôt l'objet focalisé. Devant cette incohérence, elle renonce à cette classification pour ne garder que la distinction entre focalisateur et focalisé. Genette résiste à ces catégories autant qu'à l'autonomie de l'instance focalisatrice ${ }^{13}$. Il est symptomatique que plusieurs recherches récentes, tout en reconnaissant la pertinence de l'apport balien à la théorie genettienne, continuent d'appliquer la division tripartite de Figures III. Cela tient sûrement à l'autorité de Genette parmi les spécialistes. Peut-être aussi sa typologie est-elle plus sécurisante que celle de Bal parce que davantage en continuité avec les recherches antérieures... Même la distinction au niveau de l'objet focalisé (perceptible ou imperceptible) n'a pas semblé rallier les chercheurs malgré sa cohérence.

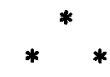

Le détour a peut-être paru un peu long au lecteur, mais je ne pouvais appliquer la notion de focalisation à un corpus sans indiquer de façon nette mes postulats et mes allégeances. Serge d'entre les morts est l'évocation de souvenirs du narrateur. Le déroulement même de la mémoire impose donc au narrateur un ordre apparemment décousu: les distorsions temporelles (anachronies) représentent ici la règle ainsi que l'a montré Bessette dans le Semestre en reconstituant l'ordre chronologique de la diégèse ${ }^{14}$. Les quarantecinq segments ou lexies ne suivent donc pas un ordre linéaire et les transitions sont assurées par un raccord syntaxique souvent astucieux où la continuité de la mémoire pallie les hiatus entre les séquences. Il est essentiel de noter la récurrence de deux moments de la diégèse: le mariage de Colette donne lieu à huit segments différents, segments tous "complétifs" 15 et ce qui entoure la mort du père s'étend à douze segments ${ }^{16}$ dont trois sont partiellement répétitifs. Je reviendrai à cette répétition significative. Il faut tout de même souligner aussi que cette récurrence correspond aux événements obsédants du premier segment (donc de l'ouverture du texte): d'abord la mort du père (p. 10), ensuite le mariage de Colette (p. 11), le tout couronné par la figure spectrale de la grand-mère ( $p .11$ et suiv.).

Première étape de mon analyse: par la voie inductive, vérifier à la fois la pertinence du cadre théorique et la complexité du corpus. D'abord un court extrait: 
[ la cuisine ] où il entra comme elle venait de poser la boîte à lunch sur le coin de la table j't'ai mis des sandwichs au jambon et il marcha vers l'évier sans même la regarder ce matin-là il paraissait plus grand que nature il avait sa grosse veine bleue qui pulsait sur sa tempe il ouvrit le robinet l'eau coulait elle trouvait que ça faisait un bruit d'été et d'ailleurs c'était presque l'été on pouvait déjà sortir en robe il commençait à faire chaud et on allait bientôt voir si le petit érable que Piphane avait planté à l'automne avait pris et elle dit j'vas-t-y te faire tes tossses tout de suite?

L'italique gras démarque deux énoncés de la grand-mère alors que le reste de la citation appartient au discours du narrateur. Et voilà pour la question "Qui parle?», relativement simple ici! Le problème de la focalisation en revanche est moins limpide. Le grand-père (Piphane) est toujours vu de l'extérieur: les énoncés il paraissait et il avait sa grosse veine bleue qui pulsait sur sa tempe ne font aucun doute là-dessus. Piphane est donc l'objet focalisé, mais qui est focalisateur? Pour une partie de l'extrait, il peut s'agir du narrateur lui-même (il reste à savoir comment Serge a pu obtenir cette information, par la famille sans doute à qui la grand-mère a dû conter l'événement). Mais la mention elle trouvait désigne un focalisé imperceptible auquel la grand-mère seule a accès. Avec on pouvait sortir en robe, le sujet féminin de la perception se dévoile sans équivoque et même le prénom familier "Piphane", sûrement privilégié par Aurore, révèle le point de vue de celle-ci ${ }^{17}$. Ainsi ce court texte offre, de façon aussi complexe qu'une partition musicale, deux voix différentes et, si l'on peut dire, deux tonalités différentes.

Comme second champ d'investigation, je considérerai les récits répétitifs (selon la terminologie genettienne). C'est un cas presque unique dans ce roman: le même événement (l'arrivée de Serge chez l'oncle Lucien) est raconté deux fois et même partiellement une troisième fois. En voici la première version:

la maison de mon oncle était toute éclairée, puis il est apparu au bout du corridor, il devait sortir de sa grande chambre qui donnait sur la cuisine, il s'est arrêté à côté de moi avec un soupir et m'a ébouriffé les cheveux et a dit comment qu'ça va mon p'tit gars? puis a regardé sa sour, pis elle? elle a pas l'air trop pire? mais elle a fait des signes avec ses mains au-dessus de moi et il a hoché la tête m'a regardé avec encore un soupir, puis levant le bras vers les chambres tu le feras coucher avec Colette, puis on montait l'escalier, ma tante derrière moi, les marches craquaient sous nos pieds, et elle a dit tu feras pas de bruit pour pas réveiller Monique pis Colette

Un seul détail trahit de façon incontestable la position du focalisateur: puis il est apparu au bout du corridor. Cette perception (de même que il devait sortir) exclut automatiquement Lucien lui-même et oriente plutôt vers l'autre bout du corridor, près de la porte d'entrée, là où se trouvent Béatrice et Serge. Comme rien n'indique la position privilégiée de Béatrice comme sujet focalisateur, c'est Serge enfant qui focalise ce récit. N'est-il pas question de la 
grande chambre, ce qui renvoie bien à la perception d'un enfant pour qui le monde des adultes est toujours plus ou moins amplifié?

La seconde occurrence étant plus longue, je me contenterai d'en citer les passages essentiels:

il pensait net de nouveau, et quand il a sorti (sic) dans le corridor je sais bien qu'il a pensé je me demande ce qu'on va dire au p'tit, et j'étais là à côté de ma tante Béatrice, minuscule et perdu au bout du corridor comme un moineau dans une cathédrale, et il marchait dans le corridor il voyait bien que ma tante Béatrice avait repris ma main dans la sienne, et il pensa c'te p'tit gars-là sait pas ce qui arrive mais y va sûrement falloir qu'avant longtemps quelqu'un lui dise quelque chose, et il s'était arrêté un moment, ou pas tout à fait, à peine le temps d'une hésitation entre deux pas, il les voyait devant la porte du vestibule, éclairés de côté par la lumière rose du salon (...) puis il était parvenu tout près d'eux, l'enfant le regardait toujours, à présent Béatrice avait posé les sacs par terre, elle lui avait enlevé son casque, il continuait de regarder Lucien en levant les yeux plutôt que de pencher la tête en arrière, et sa grosse main rouge lui caressa les cheveux et il dit comment qu'ca va mon p'tit gars? et Béatrice restait là, le casque aviateur pendant de sa main au bout de sa courroie, elle aussi regardait Lucien, le regardait avec ses yeux un peu exorbités, bouche entrouverte, son rouge trop épais sur ses lèvres molles on dirait qu'elle commence à avoir une moustache, et il demanda pis elle? elle a pas l'air trop pire? et il vit la grosse femme faire des gestes au-dessus de la tête de l'enfant et il se souvint qu'il faudrait sans doute lui dire quelque chose d'ici demain ou après-demain mais que Simone allait sûrement venir le voir et qu'elle saurait lui dire ce qu'il fallait ça prend une mère pour trouver les mots, tu le feras coucher avec Colette, puis il les regarda monter, les marches craquaient sous leurs pieds, tous deux avaient encore leur manteau, sans doute s'en débarrasseraient-ils une fois sur le palier, puis ils disparurent au tournant de l'escalier, il pouvait entendre sa sour chuchoter à tue-tête, attention de pas réveiller, puis il n'y eut plus rien,

(p. 111-113)

L'enfant qui trouvait la chambre si grande tout à l'heure n'est plus que minuscule et perdu au bout du corridor comme un moineau dans une cathédrale: le point de vue est maintenant celui de Lucien. Le narrateur est mal à l'aise dans sa fonction puisqu'il utilise fréquemment la première personne (jouant aussi un rôle comme personnage) dans cet épisode où il est focalisé par Lucien. L'énoncé je sais bien qu'il a pensé traduit ce malaise: comme Lucien focalise, Serge narrateur doit absolument savoir ce que Lucien a pensé, mais comment l'a-t-il su sinon parce que l'énoncé est vraisemblable, donc prévisible? Aussi dans ce type d'occurrence, le focalisẻ imperceptible ne peut jamais dépasser beaucoup les limites du vraisemblable. L'on comprend alors que, devant cette impasse, le narrateur préfère avoir recours à une tricherie pronominale: il les voyait devant la porte du vestibule, puis il était 
parvenu tout près d'eux, l'enfant le regardait toujours. La focalisation est donc si bien impartie à Lucien que «l'enfant» est vu comme un objet extérieur au sujet narrant. Pourtant le lecteur ne perçoit aucun hiatus: l'enfant ne vient-il pas d'être déplacé, "arraché », comme un objet justement? C'est en tout cas le souvenir qu'en a le narrateur lui-même et l'on retrouve ici par un biais étonnant la cohérence qui semblait tout à coup manquer au texte: ce qui apparaissait comme une faute technique ne serait-il pas en fin de compte un atout?

Le troisième segment répétitif raconte le même événement de façon tout à fait insolite:

et elle restait là, juste à la place où elle avait enfilé sa jaquette, écoutant encore comme pour drainer et filtrer tout ce que la maison pouvait contenir de silence ou d'autre chose, car il y avait bien quelque chose, ou quelqu'un dans l'escalier, petits bruits de souliers dans les marches, l'oreille encore fine elle entendait tout à travers le battant de la porte, puis chuchotements, attention de pas réveiller, et elle écoutait toujours,

La grand-mère, qui s'est littéralement emmurée vivante dans sa chambre (l'épisode du store cloué), s'est par le fait même condamnée à la seule perception auditive. L'arrivée de Serge se résume pour elle à du bruit dans l'escalier et des «chuchotements", attention de pas réveiller. Cette phrase-clef (voir p. 113 et 143) qui ne jouait qu'un rôle ponctuel dans les autres occurrences devient ici un élément capital pour la grand-mère focalisatrice.

On a pu mesurer, à travers cette courte enquête, l'ampleur des problèmes posés par ce texte à propos de la focalisation. Des problèmes? A vrai dire, il s'agit surtout d'un problème central déjà soulevé par Bessette dans le Semestre: par quel canal le narrateur détient-il son information sur certains focalisés imperceptibles (pensées, sentiments, impressions de la grand-mère ou de Lucien par exemple)? On accepte plus facilement l'omniscience d'un narrateur hétérodiégétique (omniscience néanmoins arbitraire), mais on s'attend, avec le narrateur homodiégétique, à ce que prédomine son point de vue ou du moins celui du personnage qu'il est dans son récit. Si un autre personnage que le personnage-narrateur occupe la position focalisatrice, les modalisateurs permettent de respecter la vraisemblance comme c'est le cas dans l'Incubation de Bessette lorsque le narrateur évoque l'univers intérieur de Néa, Gordon ${ }^{18}$, etc. Ici, un récit de Serge focalisé par la grand-mère (p. 47) commence par cela avait dû fulgurer où le modalisateur verbal présente le récit comme dominé par une conjecture. Mais c'est là une exception dans ce roman et l'ensemble des passages non focalisés par Serge (narrateur ou personnage) n'est pas marqué par la modalisation. Quels sont ces autres focalisateurs? Lucien (p. 25, 55, 110 et suiv., 125 et suiv., 133-134), la grandmère (p. 47 et suiv., 82 et suiv., 104 et suiv., 133 et suiv.), le père (p. 71-72, 136 et suiv.), Béatrice (p. 109), Colette (p. 50) et la mère (p. 35-36). Ces deux dernières occurrences méritent quelques mots de commentaires. Dans le premier cas, c'est Colette qui découvre la grande flaque malodorante sous la chaise de sa grand-mère. Le texte mentionne mais elle ne pensait plus à la 
couverture, accédant ainsi aux pensées de Colette. La mention étant bien courte, cela n'infirme pas vraiment l'opinion de Bessette sur l'absence du point de vue de Colette dans le roman. On peut en dire autant de la mère sur laquelle Bessette revient quelquefois (entre autres aux pages 155 et $203 \mathrm{du}$ Semestre). Le passage évoqué plus haut (p. 35-36) ne fait aucun doute quant à son sens, mais il est bien l'exception qui confirme la règle voulant que l'optique de la mère ne soit pas représentée: avec un frisson de dégoût, pourquoi Lucien tenait-il donc à conserver cette relique atroce, elle ne comprenait pas, elle sentait, ah cela lui donnait envie de cracher par terre. Ce court épisode contient tout de même une série de focalisés imperceptibles de la mère; de plus, il concerne les fameuses dents de la grand-mère, objet symbolique s'il en est auquel j'aurai l'occasion de revenir.

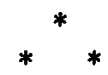

S'il se produit pareil étalement de la focalisation chez les personnages de ce roman, c'est peut-être qu'une même thématique tend justement à disséminer ses motifs tout au long du parcours. J'ai déjà fait allusion à l'importance des perceptions dans Serge d'entre les morts: il faudrait s'arrêter en particulier à la vue et au regard. Un simple coup d'œil (voilà que je focalise aussi!) même superficiel sur le lexique du roman révélera la fréquence élevée des lexèmes de la vision, entre autres "voir» et "regarder":

il ne voulait absolument pas avoir vu, discrétion, comprenait qu'il fallait bien que je fasse mes premières expériences, s'exercer tout seul dans son coin, et je pensais un bon jour il faut que je m'arrange pour la voir pendant qu'elle l'ôte, je la guettais hypocrite, elle marchait et moi je regardais Tantale ses jambes ses chevilles un peu fortes je les aimais et des fois ses jambes étaient nues c'était l'été elle portait des robes de coton comme les petites filles mais c'était déjà une fermme, une vraie femme, ses seins ses jambes, obsédé louchant sous sa robe, trucs subtils pour tâcher d'apercevoir, ah parfois blancheur de culotte dans la pénombre secrète de. viande et toison c'était l'été! la voir oui la voir au moins une fois quand on peut voir ses jambes jusqu'en haut, pas en maillot de bain, non, culotte blanche un peu moite c'était facile à imaginer, moite avec ses odeurs, me cacher m'infiltrer quelque part, à moi tout seul les vieillards de cette Suzanne, clandestin en embuscade, toute l'âme remontée dans les yeux, ni vu ni connu voir comment, et comme un fou je regardais les jambes de Colette, tu devrais porter tes jupes plus longues, elle marchait dans le corridor c'était l'été et elle portait des petites sandales de cuir et je voyais encore ses jambes et je pensais à ses pieds et j'imaginais le reste et je me suis tapi dans son placard

(j'ai souligné les termes de vision)

Cet extrait est représentatif du roman, encore que la récurrence de certains lexèmes soit ici presque extrême (même les clichés «lorgnent" du bon côté: $n i$ vu ni connu). Cependant, certaines expressions plus spécialisées comme louchant sous sa robe, clandestin en embuscade, etc., ainsi que la référence à 
l'histoire biblique de Suzanne et des vieillards, orientent la lecture, de façon plus spécifique, vers le thème du voyeurisme (l'épisode du placard évoqué ici et développé ailleurs, soit aux pages 23-24 et 65-67): mais ce thème commande davantage le choix du verbe «regarder» que celui de «voir». Le texte modulera sur cette distinction: parfois on verra sans regarder, l'action de voir étant quasi automatique (p. 74 et 83); plus souvent, on regardera mais sans réussir à voir, soit à cause de l'éclairage, soit par un manque de concentration (p. 55 , $73,98,109-110,126$ ). Serge, le narrateur et protagoniste, ne sera jamais pris en défaut lorsqu'il s'agit d'épier. Il sera plutôt pris en défaut pour avoir trop épié comme dans la scène du placard (p. 66), alors que d'autres fois il procédera plus discrètement (p. 29,62,80). Peut-être en définitive est-ce le voyeurisme même du personnage-narrateur qui rend compte de ce qu'on a appelé son omniscience. Si le voyeur ne peut voir, il fantasme: son propre cinéma intérieur lui permet de combler les lacunes de son information. On n'a qu'à considérer la scène amoureuse entre Colette et son mari pour mesurer l'aspect palliatif du fantasme: je me disais, extravoyais son sourire dans l'étreinte (p. 20), je m'imaginais facilement ce qui devait se passer (p. 29). Serge décrira la scène avec la minutie et la précision d'un observateur: je voyais ça comme si j’y étais (p. 20) et davantage encore: moi je les voyais comme si j'avais été couché avec eux (p. 100). Ce poste d'observation privilégié (aux premières loges) est peut-être le même que celui du témoin indiscret des ébats des grands-parents (p. 97-98). En tout cas, il semble bien qu'il faille postuler ici l'existence d'une instance focalisatrice extérieure aux deux personnages, instance qui voit Piphane regarder Aurore. De ce point de vue, ce passage diffère totalement du monologue intérieur de la grand-mère (p. 84 et suiv.), seule focalisatrice de ce récit de leurs activités sexuelles. Mais par rapport à l'omniscience du narrateur, le problème des deux extraits est le même. Cette troisième personne (invisible) qui observe les grands-parents n'en évoque-t-elle pas une autre, celle qui assiste ou croit assister à la Scène entre les parents:

Scène originaire. Scène de rapport sexuel entre les parents, observée ou supposée d'après certains indices et fantasmée par l'enfant. Elle est généralement interprétée par celui-ci comme un acte de violence de la part du père. ${ }^{19}$

Bessette a déjà souligné dans le Semestre l'importance de la scène originaire dans le roman de La Rocque, celle que l'enfant Serge perçoit lors d'une dispute entre les parents juste avant la mort du père. Colette et la grand-mère étant chacune à sa façon un substitut de la mère (Colette est l'objet sexuel interdit et la grand-mère, la mauvaise mère) ${ }^{20}$, on comprend mieux alors que la véritable scène (non décrite) soit éclatée et déplacée (en termes psychanalytiques): c'est la scène de découchage ou de couchage (Serge enfant couche avec Colette) et surtout la scène, plus explicitement sexuelle, entre les grandsparents. Dès lors, la répétition compulsive (véritable bégaiement narratif) du narrateur s'explique mieux aussi: l'arrivée de Serge chez Lucien (analysée précédemment) est racontée trois fois car cet épisode est l'événement-charnière entre les deux Scènes, la Scène entre les parents et la Scène de l'inceste symbolique avec Colette. Serge s'est senti arraché (p. 143, aussi p. 70 et 79) à sa maison (ce symbole de la mère est bien connu): il éprouverà le sentiment 
de rejet où l'on croit que l'autre ne s'intéresse pas assez à soi. “Regarder» signifie alors pour l'enfant "chercher l'attention (le regard) de l'autre»; "ne pas regarder" dans le cas du parent est synonyme de "ne pas s'intéresser à»:

dans les yeux mon regard planté de loin dans ses yeux et pensant tu vas me regarder il faut que tu me regardes, impression que mes yeux étaient des projecteurs, pouvoir l'obliger à, (...) je voyais aussi la face rouge de Lucien et je pensais ça y est elle m'a oublié elle ne me voit plus.

Il s'agit ici du remariage de la mère et le besoin névrotique du fils ne fera ensuite que reproduire avec Colette le même schéma: je la regardais (...) elle ne me regardait pas (p. 22-23). Les exemples sont nombreux où Serge cherche désespérément du regard l'attention de Colette (p. 30, 62, 120, etc.). Il s'habituera ainsi peu à peu à l'absence de réciprocité: l'impossible à aimer autrement que du fond des yeux (p.65) deviendra celle dont on doit éviter à tout prix le regard (ne pas me faire voir mais ne pas la perdre de vue non plus, p. 80). De la larme à l'œil on passe aisément à l'arme à l'oeil! Voir par tous les moyens ! Pourtant, l'interdit demeure: défense de regarder Gorgone !... je ne pensais presque plus à Colette (p. 94). On sait ce qui arrivait aux malheureux qui osaient défier le regard de Gorgone: ils étaient pétrifiés comme l'avaient été la femme de Loth dans la Bible. On ne s'étonnera donc pas du fait que la grand-mère, qui pouvait voir briller les yeux voyeurs de Piphane (p. 12), qui a décidé d'en finir avec la chose (c'est assez c'est fini la paix la paix la paix, p. 86) et avec la vue de la chose (la paix la paix la paix, p. 15), soit une obscure divinité statufiée, ah oui statue inexorable (p. 16), qu'elle se minéralise (p. 18), qu'elle soit granit des pieds à la tête (p. 83), que son cerveau soit pétrifié (p. 134). Pétrifiée donc pour avoir trop regardé, ou pas assez, la grand-mère s'est elle-même condamnée à ne plus voir, à être le complément parfait de Serge voyeur, l'oreille tendue comme l'enfant qui ne peut voir la Scène et qui en est réduit à la fantasmer à partir des bruits. Il est donc logique que l'aïeule incarne l'aspect auditif de la Scène, les grincements de la chaise berceuse comme les ressorts d'un lit, ce qui s'oppose fortement pour l'enfant aux bruits de père et mère avec leurs souliers ( $\mathrm{p}$. 146), bruits rassurants parce que non sexuels et n'impliquant donc pas pour lui de choix crucial (p. 41, 63, $90,116)$.

Cette figure de pierre, ce masque impassible (p. 83) est une morte-vivante précisément parce qu'elle ne voit plus rien: store perpétuellement baissé il n'y a plus de vie. Non seulement elle s'est privée de tout spectacle, mais elle ne regardait rien ni personne elle avait des yeux d'animal empaillé (p. 39). Mais ce "masque» sans expression, c'est aussi l'absence de visage ou le visage du parent que l'enfant n'arrive pas à reconnaître, qu'il ne veut pas reconnaitre. Le thème de la scotomisation du visage parental doit ici être évoqué: refus pour l'enfant de regarder les organes sexuels des parents qui sont alors fréquemment l'objet d'un déplacement (le haut pour le bas) ${ }^{21}$. C'est justement parce qu'elle veut ressembler à votre mère que la face de cire blanche de la femme rouge est si terrifiante (au musée de cire les personnages si ressemblants si réels qu'on avait envie de toucher pour voir si, p. 126). Cette mère 
qui reste penchée comme pour ne pas me regarder ou pour que je ne puisse pas voir sa face (p. 89). On peut alors superposer plus aisément l'immense bouche rouge barbouillée de rouge à lèvres rouge très rouge (p. 145) de la femme à la face de cire blanche des cauchemars de Serge et la bouche rouge lubrique, sang on dirait comme pour être déflorée (p. 20 - je souligne) de la Colette fantasmée par Serge. Colette et son ami qui flicflaquaient (p. 20) ne font pas autre chose que Piphane et Aurore: c'est comme flicflac quand je bats des aufs (p. 86). Secret culinaire transmis par l'ancêtre? Peut-être bien... A moins que ce ne soit toujours la même Scène que se représente Serge! Même représentation pour le visage du père qui, tout en étant bien conservé, ne se ressemble pas:

et jai revu longtemps et je vois encore comme autrefois sa face par miracle préservée sa face exsangue et comme souriante par défi dans le cercueil je voulais l'embrasser je voulais poser mes lèvres sur cette chose froide qui en fait ne ressemblait plus vraiment à mon père ni à rien qui avait pu vivre ailleurs que dans mes souvenirs et dans les rêves que je ferais et dans les apparitions blanches que je forcerais à surgir d'entre les ombres chaque fois que je prononcerais ou écrirais son nom ou que j'évoquerais les contours vacillants de son visage

je le revois sa face cireuse exagérément maquillée par d'impudiques croque-morts pour faire dire ah mon Dieu qu'il se ressemble! mais moi je pensais c'est pas lui c'est un autre

Ailleurs le père ne se ressemblait plus ce n'était déjà plus que viande (p. 41). Le choix du lexème «viande» paraît contredire les autres mentions (exsangue, cireuse) encore qu'il rejoigne bien l'expression la peau bleutée d'une dinde (p. 42). Il faut observer que viande dans le roman a souvent le sens de chair désirable (p. 23, 51,61, 65, 66, 120, 122) ou d'organe sexuel (p. 85), ce qui confère à ce lexème le même statut que la bouche rouge.

Ainsi le rapport à la scène originaire rendrait compte du thème du voyeurisme dans Serge d'entre les morts. Peut-être est-ce pour cette raison que même le travail de Serge comporte un caractère punitif (et corrélatif à la faute):

éclaboussant toute la shoppe d'une lumière d'enfer qu'il ne fallait pas regarder sous peine de se retrouver la nuit suivante avec des tonnes de sable sous les paupières, abrasif on aurait dit dans les yeux, dans l'obscurité voir partout des projecteurs jaunes et des centaines d'éblouissements de magnésium, fallait mettre des poches de thé mouillées, compresses que ça se calme, défense de regarder Gorgone!... (...) l'autre jour le bonhomme qui travaillait à la plieuse s'est fait crever un oeil, comme jaune d'œuf je le jure!

Il ne reste plus d'autre solution pour le petit-fils que d'être à l'affût comme sa grand-mère, les oreilles dans le crin. On a pu constater que, si Serge est le 
principal sujet focalisateur et voyeur, il n'en a pas le privilège exclusif (un auteur n'est pas tenu à s'identifier au seul narrateur fut-il homodiégétique). Il demeure que c'est lui qui est au centre de la parole et de la vision (ou perception). Ce sujet qui n'en finit plus de regarder s'est lui-même isolé; n'ayant pu épuiser l'objet du regard, il s'épuisera maintenant dans l'autoérotisme: éternité du geste lorsque votre poignet vous aime (p. 120). L'expression, stupéfiante à prime abord, s'éclaire singulièrement si on la rapproche de son visage m'aimait (p. 116). L'identité de structure est frappante: dans les deux passages, c'est un objet qui aime (Serge ne dit pas «ma mère m'aimait», mais son visage m'aimait). On peut présumer que les deux objets jouent un rôle comparable auprès de Serge. Colette, l'impossible à aimer autrement que du fond des yeux pourra devenir celle qu'on aime du bout des doigts ou mieux, à pleines mains (p. 67 et 121). Iconicité du premier extrait (p. 67) où l'ellipse de la page 66 (il fallait que je) correspond bien à la suppression (répression) au plan du contenu; il faudrait plutôt parler de suspension puisque la phrase sémantique se termine de fait à la page 67: ça ruisselait spasmodique. Quand au second passage, je me permets d'en citer quelques lignes:

j'ai arrêté ma main, ça sortait de mon poing c'était tout étranglé, comme foie de poulet, je la serrais si fort que je devais avoir l'air d'un gars en train de tirer au poignet, des histoires pour me l'arracher, m'équeuter aussi sec, l'instinct à ce moment-là!

Citation qui en sollicite une autre:

je les regardais au fond de ma main et je sentais comme une chaleur étrange me monter dans le poignet et dans l'avant-bras, comme la sensation de tenir entre mes doigts non seulement la relique mais aussi la sainte dépouille elle-même, sa mâchoire dans ma main, cette espère de protubérance je dirais informe et à peu près cartilagineuse qui bougeait perpétuellement autour de la paparmane, oui j'avais l'impression de la serrer dans ma main et de tordre et d'arracher et de briser sec.

La serrer dans ma main: phrase grammaticalement correcte puisque "la» désigne bien la mâchoire. Et pourtant! Pourquoi cette chaleur étrange qui lui monte dans le poignet comme cela se passe dans la scène masturbatoire de la page 121 ? Et la reprise quasi identique de arracher et de briser sec dans me l'arracher, m'équeuter aussi sec: Serge voudrait-il parler du même objet? Le terme "équeuter" évoque le châtiment de la castration (ou le sentiment de culpabilité du sujet qui craint la castration). Serge ne raconte-t-il pas dans la même page l'histoire de Johnny dont le père castrateur, l'ayant surpris à se masturber, lui avait donné une claque majuscule à lui arracher la tête (et quelle tête?)? Dans l'épisode du placard, contexte où la masturbation et le voyeurisme se conjuguent parfaitement, le narrateur ne fait-il pas allusion à un chien à grosses mâchoires qui vous castre d'un coup de dents? Comparaison qui ne donne pas le change... La principale figure castratrice du 
roman demeure la femme rouge avec ses longues dents blanches (p. 145) ou son équivalent, la grand-mère: il n'est donc pas étrange de retrouver ici (à la page 42) les dents de l'aïeule! De plus, la récurrence du terme "arracher» polarise l'attention du lecteur. Quelquefois, il s'agit par là de s'arracher à la maison, à l'emprise familiale (p. 70, 79, 143 - la maison était là, vivante et mangeuse, p. 141). Mais pour d'autres occurrences comme celle de la page 42, ne doit-on pas se rappeler aussi que l'arrachage des dents peut être, selon la psychanalyse, un symbole de masturbation (et, bien sûr, de castration punitive) 22 ? Ainsi le parallélisme entre les deux passages confrontés plus haut se comprend mieux de même que se lit plus clairement le passage où la mère va ranger les fameuses dents : elle sentait qu'elle avait besoin de se laver les mains, un peu comme un enfant qui vient de se masturber (p. 36). Une fois de plus, une simple comparaison se révèle rien moins qu'un ornement.

La figure castratrice appelle une revanche du sujet castré (ou à castrer) dont l'agressivité donnera libre cours à ses pulsions violentes. Regard de Serge qui n'est pas que caresse: je la regardais avec mes dents (p. 24). Façon particulièrement incisive de regarder! On doit donc entendre toute la littéralité des propos du narrateur lorsqu'il mentionne: les garçons à face de mouche la mangeaient des yeux (p.65). Serge narrateur pourra aussi fantasmer, à propos de sa grand-mère, que son grand-père la mordit dans le cou (...) et la reprit avec une fureur nouvelle, profondément comme s'il avait voulu la clouer par le dessus du crâne au bout du lit (p. 97-98). Le clouage n'est pas ici simple métaphore: la violence con'notée répond bel et bien à l'évocation de la page 108 (le gars à qui le bourreau est en train de clouer les testicules au bois de sa chaise avec des clous rouillés). Tel grand-père, tel petit-fils! Serge pourra dire d'une partenaire d'occasion: j'aurais pu la clouer raide là sur le sol comme siffleux d'un coup de fourche j'étais mâle suprême (p. 124). La dernière mention renchérit sur l'acte de clouage en soulignant le rapport de domination. De plus, la figure du coup de fourche doit se lire dans toute sa polysémie (juteuses les fourches, p. 29, lui baver dans la fourche, p. 94, je me suis lavé la fourche, p. 101). Violence des fantasmes du narrateur à relier à la violence de la scène originaire telle que perçue par des indices (p. 72) ou telle que fantasmée (p. 97-98). C'est le viol lui-même qui représente l'état maximal de ces pulsions violentes: je la regardais dans les yeux comme pour au moins la violer dans sa face et dans sa tête (p. 120), la violer à corps perdu devant tout le monde (p. 59). Il faudrait surtout s'arrêter à un texte impliquant le même fantasme, mais chez la grand-mère cette fois:

oui y a quéqu'un dans l'escalier j'suis sûre qu'y a quéqu'un dans les marches ! encore des grincements, ou un craquement plus fort peut-être - ou même rien de cela, probablement la seule certitude qu'IL était là et qu'IL restait sur les marches et peut-être montait à sa chambre dans la pénombre pour l'assassiner ou même la violer affront suprême jetée sur le lit d'un seul coup de poing au visage et tout étourdie sentant horreur de grosses mains d'homme écarter ses cuisses maigres et le narf honteux la pénétrant et allant et venant dans l'immémoriale plothe sèche comme un trou de noud dans une planche de bois franc et le couteau qui lui tranche 
méticuleusement la gorge ah mourir ! aux aguets elle savait que ce n'étaient pas les bruits de ceux qui marchent dans les murs, qui c'est qu'est là?

On reconnaît la suite du texte déjà cité à propos de la focalisation auditive de la grand-mère. L'ensemble de cet extrait est imputable au narrateur sauf un énoncé en italique (qui c'est qu'est là ?), l'italique désignant le discours direct, et un autre énoncé sans italique (oui y a quéqu'un dans l'escalier j'suis sûre qu'y a quéqu'un dans les marches!) représentant un monologue intérieur comme dans le long passage des pages 84 à 88 , monologue démarqué dans ce cas par une parenthèse. Et pourtant, malgré l'attribution de la parole à Serge, c'est bien la grand-mère qui focalise comme en témoignent les expressions sentant horreur de grosses mains d'homme écarter ses cuisses maigres et narf honteux (je souligne). Le syntagme narf honteux en particulier apparaît complexe: «narf» renvoie par sa prononciation à la langue populaire et appartient vraisemblablement au "petit lexique" de la grand-mère encore que, par l'absence de guillemets, le narrateur le prenne aussi à son compte, de même que le terme «honteux", impliquant un jugement moral et la sanction d'un Surmoi. Mais qui a honte? Le sujet focalisateur sans doute, puisque "narf honteux " se retrouve dans un monologue intérieur d'Aurore (p. 85), alors sujet de parole et de focalisation à la fois; le narrateur aussi, qui semble faire corps avec le sentiment de culpabilité du personnage. Même la focalisation ne relève pas toujours ici de la grand-mère: l'immémoriale plothe sèche l'exclut probablement comme sujet de perception. Passage des plus ambigus donc, passage stratégique aussi qui est peut-être, pour reprendre Philippe Hamon, un foyer idéologique ${ }^{23}$. Car c'est l'aspect stratégique de ce court texte qui explique le mieux le brouillage des responsabilités des instances. En bref, le fantasme de viol est ici intériorisé par un sujet féminin (la grand-mère focalisatrice) sans que dans l'ensemble le narrateur ne cède la parole au personnage. Cette distorsion fait que le narrateur a tout l'air de prendre ses distances vis-à-vis de ce qui est un fantasme de femme. Caution subtile pour entériner une idéologie implicite du viol (Tout le monde aime ça, même les femmes, et même les très vieilles femmes!). C'est ainsi que se découvre l'immense pouvoir idéologique du focalisateur dans un texte ${ }^{24}$.

Au terme de cette réflexion, la fonction d'une scène particulièrement violente et non moins insolite apparaîtra plus clairement : après une activité sexuelle avec Aline dans la chambre même de la grand-mère, Serge pillera la chambre, lancera les dents par la fenêtre et commencera même un petit incendie. Si la scène amoureuse avec Aline peut se lire comme un inceste par métonymie (cela se passe dans la chambre de la grand-mère, substitut de la mère), l'acte de destruction doit s'entendre comme un défoulement de l'agressivité contre la pulsion incestueuse elle-même et en même temps contre la figure du Surmoi (de là l'insistance sur les dents). Tout ce texte aura donc été traversé par le chassé-croisé d'Éros et Thanatos dont les signes sont ici presque interchangeables: lombrics la licher subito! (p. 11) et elle me lichait (p. 61); flac! floc! dégouliner maniaque dans les poils (p. 29) et hypocrite cadavre, se liquéfier ça presse ! pas moyen de tourner le dos, en profite, flatch ! je dégou- 
line !... (p. 50); il ne se ressemblait plus ce n'était plus que viande (p. 41) et quand elle courait c'était viande dessous (p. 51); d'impudiques croque-morts (p. 53) et c'était mou impudiquement mou à travers le tissu (p. 61); oui je savais que le coeur de la maison avait cessé de battre (p. 11) et mon coeur battait fou dans mes culottes (p. 123). Plaisirs de Thanatos et douleurs d'Éros!

Je ne voudrais pas conclure en donnant l'impression que ces propos sont exhaustifs : parcourir une allée de jardin n'est pas parcourir tout le jardin ! On pourrait suivre aussi d'autres pistes de lecture : temps et mémoire par exemple ou ce que le narrateur appelle son cinéma olfactif ou enfin les procédés d'écriture dont certains ont été repérés avec justesse par Gilles Dorion dans Après la boue ${ }^{25}$. Ce que j'ai tenté, à partir d'un thème central de ce roman, c'est de démontrer la profonde cohérence de ce texte, malgré des incohérences ponctuelles comme les fautes d'optique relevées par Bessette. C'est cette cohérence textuelle, cette lisibilité, qui rend anodines aux yeux du lecteur les fautes mineures. Le tandem scène originaire - voyeurisme explique la frénésie du discours du narrateur: on pourrait dresser la liste des marques stylistiques de ce discours, (par exemple l'hyperbole, l'accumulation, l'ellipse, l'anacoluthe, etc.). Discours fébrile donc parce qu'il retourne aux origines, qu'il ne craint pas d'avoir recours aux "popa» et aux "moman" de notre enfance québécoise, qu'il fait surgir Serge et la Scène d'entre les morts (la référence à l'intertexte biblique «ressuscité d'entre les morts» souligne dès le titre la primauté du visuel). Fébrilité du narrateur qui sait aussi que, sur la scène originaire, veillaient des yeux et des oreilles appartenant à des censeurs représentant Dieu et la Famille (p. 28). Après tout, ce sont peut-être les premiers voyeurs.

1. Gilbert La Rocque, Serge d'entre les morts, Montréal, VLB éditeur, 1976.

2. Femmes imaginaires; l'Ancien Testament au risque d'une narratologie critique, Montréal, Hurtubise HMH, coll. «Brèches", 1985.

3. Gilbert La Rocque: l'écriture du rêve, Montréal, Québec/Amérique, 1985, p. 45.

4. Montréal, Québec/Amérique, 1979. Sur la fascinante parenté entre Bessette et La Rocque telle que révélée par Bessette lui-même dans le Semestre, il fáut lire le texte de Réjean Robidoux "Gérard Bessette, lecteur de Gilbert La Roche", dans Gilbert La Rocque: l'écriture du rêve, p. 89-102.

5. Ibid., p. 198 et suiv.

6. Paris, Éditions du Seuil, coll. «Poétique», 1972, p. 203 et 206.

7. Paris, Editions du Seuil, coll. "Poétique», 1983, p. 43.

8. "Narration et focalisation", Poétique, 29, février 1977, p. 119-120. Ces vues sont reprises dans "The laughing mice or: on focalization", Poetics Today, 2, 2, winter 1981, p. 201-210, et dans Femmes imaginaires déjà cité.

9. Paris, Klincksieck, 1977 , premier chapitre; ce chapitre reproduit à peu près intégralement l'article «Narration et focalisation».

10. Nouveau discours du récit, p. 48 en particulier. 
11. A titre exemplaire, revoir Roland Barthes, «La mort de l’auteur», dans le Bruissement de la langue: essais critiques IV, Paris, Éditions du Seuil, 1984, p. 61-67.

12. "Le discours attributif et le récit", Poétique, 35, septembre 1978, p. 305-313.

13. Nouveau discours du récit, p. 48. Il est dommage que Genette se soit enfermé dans une position défensive vis-à-vis de ce qu'il considère comme son fief. Il n'a donc pu éviter le parti pris dans sa réponse à Mieke Bal (Nouveau discours du récit, p. 50). Cela se révèle même dans sa bibliographie où Bal n'est pas citée selon les règles d'usage! En revanche, cette dernière dans Femmes imaginaires (p. 65 et suiv.) maintient la polémique à un plus haut niveau, en ajoutant même des arguments inédits.

14. P. 19 et suiv. La reconstitution chronologique d'un roman rempli d'anachronies s'avère souvent utile, pour ne pas dire indispensable, au travail interprétatif. Dans l'ensemble, je souscris au découpage effectué par Bessette. Je me permets cependant quelques divergences d'opinion: la scène VIII - scène du papillon (le Semestre, p. 28) se situe peu de temps après le remariage (Serge, p. 32) et Serge n’a donc que cinq ans environ et non dix; les scènes $X, X I$ et XII sont presque impossibles à distinguer au plan chronologique; enfin, la scène des dents devrait plutôt suivre la scène XVII (copulation avec une inconnue) si l'on en croit l'information donnée à la page 70 .

15. Voir p. 19-20, 21-23, 26-28, 42-43, 59-62, 99-102, 139-143, 146-147.

16. Voir p. 40-42, 52-53, 54-55, 55-56, 71-73, 82-84, 107-115, 116-117, 117-118, 133-136, 143-146.

17. L'utilisation inégale des prénoms des grands-parents est significative à cet égard: "Aurore» ne revient que deux fois (p. 97- et 103) alors que «Piphane" est nommé à maintes reprises dans le roman. Ce traitement inégal, révélateur de focalisation, apparaît dans toute sa netteté à la page 142 oủ le narrateur parle de «Piphane» et de "grand-mère" dans la même phrase.

18. On pourra consulter à ce sujet mon analyse dans Gérard Bessette: l'Incubation et ses figures, Montréal, Presses de l'Université de Montréal, 1983. p. 164-165.

19. J. Laplanche et J.-B. Pontalis, Vocabulaire de la psychanalyse, Paris, Presses Universitaires de France, 1984, p. 432.

20. Sur ce thème déjà traité judicieusement par Bessette dans le Semestre, on relira avec profit l'excellent texte d'André Vanasse "La fête, la haine, la mort", dans Gilbert La Rocque: l'écriture du rêve, p. 103-121.

21. Mon information sur ce thème provient de mon collègue et ami Henri-Paul Jacques qui, en plus de me fournir ses propres textes non encore publiés, m'a initié à certains éléments bibliographiques sur le sujet comme par exemple l'article de Nathan Roth "The Face-Genital equation», The Psychoanalytic Quarterly, vol. 28, 1959, p. 493-500. La figure du masque occupe évidemment le premier plan dans le roman de I_a Rocạue les Masques.

22. Sigmund Freud, Introduction à la psychanalyse, Paris, Petite bibliothèque Payot, 1975, p. 142.

23. "Texte et idéologie», Poétique, 49, février 1982, p. 105-125.

24. Cette avenue est abondamment explorée dans Femmes imaginaires de Mieke Bal déjà cité. L'aspect idéologique d'un texte narratif est aussi abordé, quoique dans un cadre théorique différent, dans l'excellent article de Neil B. Bishop «Distance, point de vue, voix et idéologie dans les Fous de Bassan d'Anne Hébert", Voix et images, vol. IX, 2, hiver 1984, p. I13-129.

25. «Une rhétorique efficace " dans Gilbert La Rocque : l'écriture du rêve, p. 75-88. 